\title{
Aerodynamic Analysis of Backward Swept in HAWT Rotor Blades Using CFD
}

\author{
Mohammad Sadegh Salari ${ }^{1}$, Behzad Zarif Boushehri², Mehrdad Boroushaki ${ }^{*}$, \\ ${ }^{1,3}$ Department of Energy Engineering, Sharif University of Technology, P.O. Box 14565-114, Tehran, Iran \\ ${ }^{2}$ Department of Aerospace Engineering, Sharif University of Technology, P.O. Box 11155-11365, Tehran, Iran
}

\begin{abstract}
The aerodynamical design of backward swept for a horizontal axis wind turbine blade has been carried out to produce more power at higher wind velocities. The backward sweep is added by tilting the blade toward the air flow direction. Computational Fluid Dynamics (CFD) calculations were used for solving the conservation equations in one outer stationary reference frame and one inner rotating reference frame, where the blades and grids were fixed in reference to the rotating frame. The blade structure was validated using Reynolds Averaged Navier-Stokes (RANS) solver in a test case by the National Renewable Energy Laboratory (NREL)

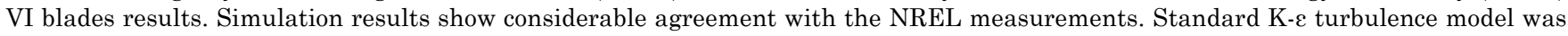
chosen for simulations and for the backward swept design process. A sample backward sweep design was applied to the blades of a Horizontal Axis Wind Turbine (HAWT) rotor, and it is obtained that although at the lower wind velocities the output power and the axial thrust of the rotor decrease, at the higher wind velocities the output power increases while the axial thrust decreases. The swept blades have shown about 30 percent increase in output power and about 12 percent decrease in thrust at the wind speed of $14 \mathrm{~m} / \mathrm{s}$.
\end{abstract}

Keywords: Horizontal Axis Wind Turbine, CFD, Backward Sweep, Wind Turbine Efficiency, Blade shape Optimization .

Article History: Received June 23 ${ }^{\text {rd }}$ 2018; Received in revised form Sept $16^{\text {th }}$ 2018; Accepted October $1^{\text {st }}$ 2018; Available online

How to Cite This Article: Salari, M.S., Boushehri, B.Z. and Boroushaki, M. (2018). Aerodynamic Analysis of Backward Swept in HAWT Rotor Blades Using CFD. International Journal of Renewable Energy Development, 7(3), 241-249.

http://dx.doi.org/10.14710/ijred.7.3.241-249

\section{Introduction}

Wind power generation is an environmental friendly method of generating electric energy through the operation of a generator attached to the axis of a rotor blades that turns due to the rotational action of the resultant aerodynamic force generated by the change of wind momentum. Since wind energy is a low-density source of power, it is of great importance to maximize the efficiency of wind machines.

Wind turbine metrics is measured by their "rated power" which can be retrieved from the incoming wind. It is typically presented in non-dimensional form by the rated power coefficient. This coefficient expresses the ratio between the retrieved wind power to the power available in the incoming wind (Betz, 2014). The maximum achievable value of the power coefficient is known as the Betz limit. According to Betz limit, the maximum power coefficient of a wind turbine is 0.593 . Typical Horizontal Axis Wind Turbine (HAWT) demonstrates a value near 0.4 (El-Wakil, 1984). Major factors of difference between this practical value and Betz limit are aerodynamical losses which mainly refer to airfoil performance, blade shape and wake generation.

The aerodynamics of flow around the wind turbine blades plays an important role on wind turbine efficiency.
Currently, there is a great interest in studying wind turbines to improve its aerodynamic performance (Farrugia et al, 2016; Subramanian et al, 2016; Sedaghat et al, 2014; Jeon et al, 2014; Li et al, 2012).

The objective of this work is to carry out flow analysis to a horizontal axis upwind wind turbine, where the rotor blades are straight at first and are backward swept thereafter. In these configurations, the effect of two parameters -wind speed and swept angle- will be investigated on turbine output power and axial thrust. This work aims to investigate detailed three-dimensional flow analysis and quantification of the resulting thrust and power output on the both straight and swept upwind moving turbine blades and exploring the effect of changing the shape of a typical straight HAWT's blade.

Computational Fluid Dynamics (CFD) analysis of wind turbine can be performed at a cost less than what is required to set up a wind tunnel and perform full scale experiments. A growing number of researchers are using computational fluid dynamics to study wind turbine aerodynamics such as: Li et al (2012), AbdelSalam et al, (2014), Janajreh et al, (2010), Cao et al, (2012), Mohamed et al (2015), Make et al (2015) and Balduzzi et al, (2016).

The Reynolds Averaged Navier-Stokes (RANS) solver and direct modeling of the rotor by constructing a body-fitted grid is used to simulate the proposed blade

\footnotetext{
* Corresponding author: +98-21- 66166102 (boroushaki@sharif.edu)
} 
shape. Body-fitted grid is particularly suitable for internal or external flows with smoothly-varying nonregular boundaries. In order to model a complex geometry, we divide the flow region into various smaller subdomains. All these regions have been meshed separately and joined up correctly with the neighbors.

It is noteworthy that backward swept has been already added to an aircraft wings for the transonic cruise at Mach numbers where shock waves are present. (Chattot, 2009). The backward sweep is chosen in general since it is a more structurally stable configuration. In addition to its effects at higher speeds, also it has a slight but good effect on aero- dynamical performance of airplanes at lower speeds.

Application of backward swept configuration in rotor blades in this study show that although at the lower wind velocities the output power and the axial thrust of the rotor decrease, at the higher wind velocities the output power increases while the axial thrust decreases.

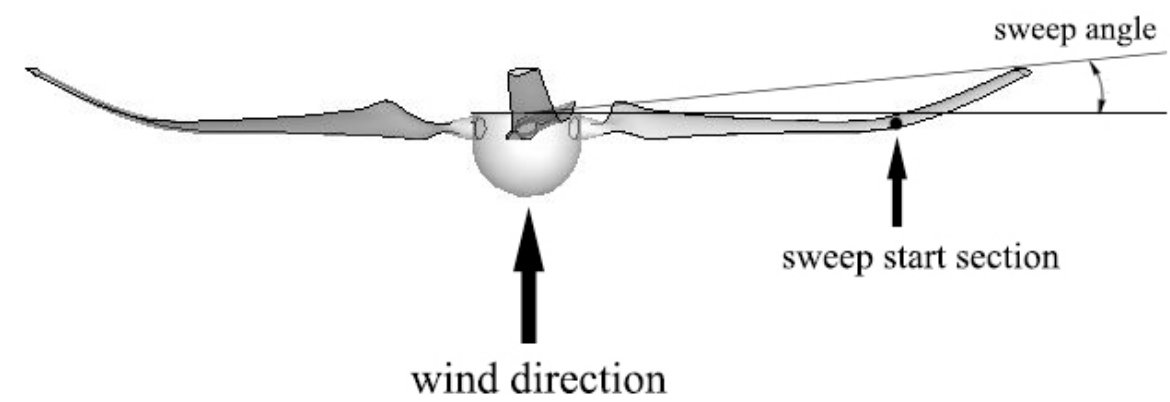

Fig. 1. HAWT rotor with backward swept structure

\section{Backward swept in HAWT rotor blades}

The physical problem considered in the present research is calculating the flow around the horizontal axis wind turbine (HAWT), first with straight blades and then continued by optimized backward swept blades as shown in Fig. 1

The testing wind turbine is the National Renewable Energy Laboratory (NREL) phase VI, a modified Grumman Wind stream 33 stall regulated turbine with full-span pitch control at a power rating of $20 \mathrm{~kW}$. Giguere et al, (1999). It has three straight blades, with NREL S809 linear tapered and nonlinear twisted blade profile. The rotor diameter is $10.058 \mathrm{~m}$ and it has a fixed blade pitch angle of 5 degrees with a constant rotational speed of $72 \mathrm{rpm}$. The experiments performed in the NASA Ames wind tunnel in 1999 are considered as a benchmark for the evaluation of aerodynamic results. Detailed geometry, machine parameters and experimental procedures can be found in the NREL report (Giguere et al, 1999).

\subsection{Computational domain}

Fig. 2 shows the computational domain considered in the CFD solver. The wind turbine rotor is located in the center of the domain, and the point of rotation is considered as the origin point. The rotor has a diameter of approximately 10 meters and rotates at $72 \mathrm{rpm}$. The S809 series airfoils are used for modeling the blades. The domain considered for analysis is $10 \mathrm{D}$ in the spanwise direction, and 25D in the both downstream and upstream regions, where $\mathrm{D}$ is the diameter of the rotor.

The literature suggests that blockage ratios (ratio of model frontal area to test section area) below the range of $6-7.5 \%$ have a negligible effect on the flow, while the blockage ratio in generated mesh is close to $1 \%$ (AbdelSalam et al, 2014).

The computational domain was meshed with a mixed three-dimensional mesh to carry out the wind turbine fluid flow simulation. The mesh elements adapted for the computational model are shown in Fig. 3. In the stationary domain, the tetrahedral meshes grow in size from the interior boundary between rotating and fixed domain, toward to the outer boundary as shown in Fig. 3a. In order to resolve the strong gradients in the vicinity of the wind turbine rotor, a high concentration of grid points was distributed in the region around the rotor to account for the wake expansion as demonstrated in Fig. 3b. The surface mesh element sizes are controlled to obtain fine mesh elements close to the blades and hub.

A boundary layer mesh at 0.001-meter distance of initial thickness of 7 rows with 1.15 growth rate was generated in order to capture the viscous layer and associated viscous forces. The surface meshes were created with maps, and tetrahedral elements were used further away from the surface. The mesh grows in size outward from the rotor surface and hub to the extended domains. Fig. 3c demonstrates the general configuration of the mesh over one part of the blade surface. Different mesh configurations, starting with coarse ones to fine ones are taken at a particular Reynolds number and have been analyzed using a CFD code. The grids have been created with a grid refinement factor that has been systematically performed with a value of 1.1 .

\subsection{Solution methodology and boundary conditions}

The complete set of fluid equations consists of the continuity equation, three momentum equations for the transport of velocity, and transport equations in according to turbulence model used for $\mathrm{k}$ and $\varepsilon$. To solve 
the RANS equations in the near and far field regions of the wind turbine, the full rotor approach is applied for the representation of the rotor in the computational mesh.

The fluid equations are solved by employing a CFD code in the three-dimensional mode. It uses a control-volume-based technique for converting the governing equations into algebraic equations. The solution algorithm is SIMPLE, and the first-order upwind scheme is used for all the dependent properties. The results were extracted from the computational domain along different sections of the wind turbine blade and on the rotor.

The inlet boundary is a plane located at the upstream of the wind turbine as shown in Fig. 2. Inlet free stream velocity is considered according to the undisturbed neutral atmospheric boundary layer with constant velocity. The shear stress is assumed constant over the lower part of the atmospheric boundary layer and no-slip wall condition is assigned to the rotor blades and hub surfaces. The outlet boundary is a plane located at the downstream of the wind turbine. In this plane, the static pressure is considered to be zero relative to the atmospheric pressure. The wind turbine operates in a rotating reference frame at a constant rotational speed of $72 \mathrm{rpm}$. The interior plane is adapted to handle the interaction between the moving and fixed frames at the interface. The side walls are far enough from the wind turbine blade tips and are considered as symmetry boundary conditions. During the computations with full rotor approach, near-wall treatment for the rotor surface is handled by the standard wall functions.

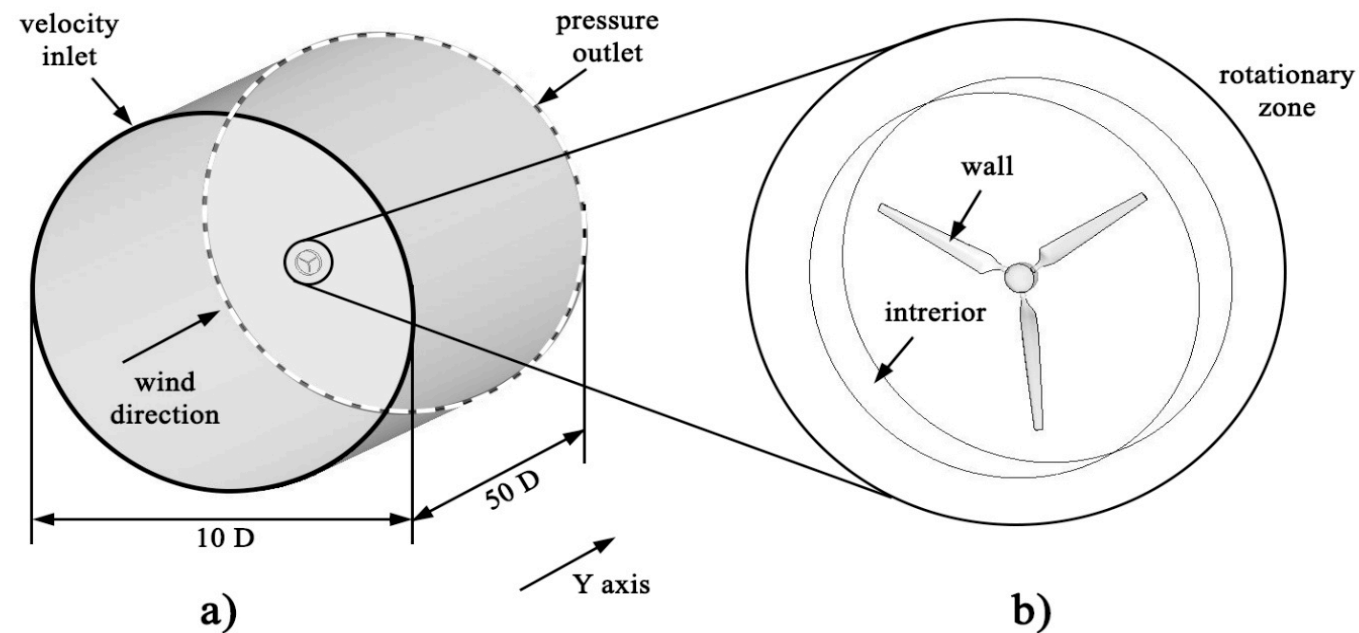

Fig. 2. Computational domain (a) Whole domain (b) Rotating reference frame

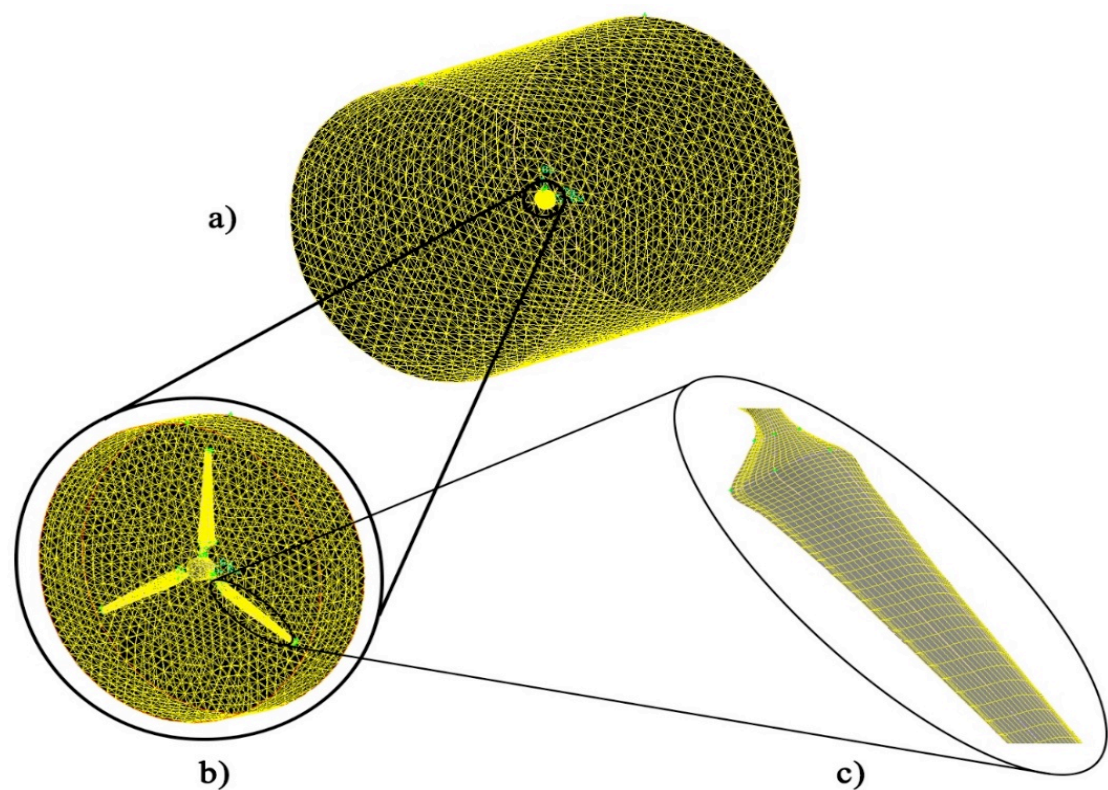

Fig. 3. Computational mesh generated (a) Typical mesh configuration over the whole domain (b) Typical mesh configuration around the rotor (c) Typical mesh configuration over the blade surface 


\subsection{Validation of the generated mesh}

In order to ensure numerical accuracy, the influence of the grid resolution has been analyzed to limit the computing cost. The method used here for grid study is to change the mesh size in order to make the required variables independent from mesh size. After finalizing the grid size, the numerical analysis is performed for the wind speeds of 4 up to $18 \mathrm{~m} / \mathrm{s}$ with steps of $1 \mathrm{~m} / \mathrm{s}$ prevailing at the hub height, using $\mathrm{k}-\varepsilon$ turbulent model. Fig. 4 indicates the results in terms of power production versus wind speeds, compared with the available experimental data to find the most convenient approach for further analysis.

After validating the results, an optimized backward sweep curve adapted to the blades of the rotor and the $\mathrm{k}$ $\varepsilon$ turbulence model was approved for analyzing the flow. The backward swept curve was implemented to the blades of the rotor by a function generates monotone Hermitian interpolants and has discontinuous second derivatives at the data points.

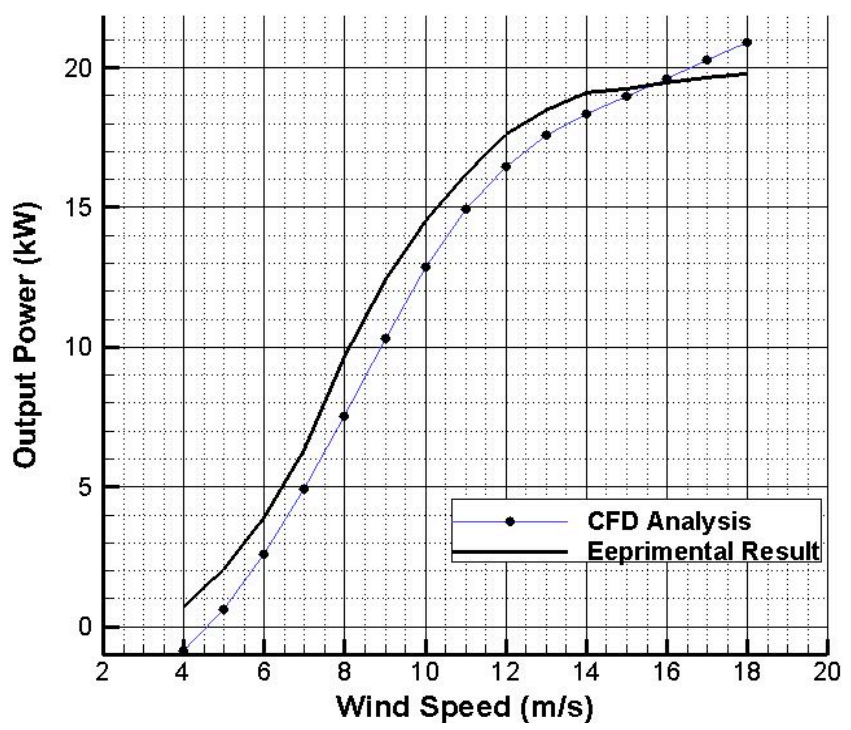

Fig. 4. Comparison of wind turbine output power versus wind speed calculated by numerical solution with experimental data

The three-dimensional steady state RANS equations have been solved using $\mathrm{k}-\varepsilon$. The results in terms of power production have been compared with the available experimental data at 12 different wind speeds. The comparison between the experimental and computed power is shown in Fig. 4.

In general, numerical solution shows considerable agreement with experimental data. At low and moderate wind speeds $(4 \mathrm{~m} / \mathrm{s}$ to $15 \mathrm{~m} / \mathrm{s})$, k- $\varepsilon$ model under predicts the actual power values, while at high wind speeds $\mathrm{k}-\varepsilon$ model over predicted. However, numerical results showed the best agreement at wind speed equal to $15 \mathrm{~m} / \mathrm{s}$ with experimental data, which is matched with the rated wind velocity in NREL case study (Giguere et al, 1999).

It should be noted, since there were not any other experimental data for NREL VI rotor such as pressure and velocity fies or other flow quantities, our validation has just limited to the comparison of power extraction between our numerical solution and available NREL experimental data. Since the aim of this paper is to design a wind turbine blade which produces more power at moderate and high wind speeds, the discrepancy occurring at low wind speeds could be of no importance.

\section{Case study: implementation of backward swept blades on NREL rotor}

The NREL phase VI rotor blade was selected to be the baseline blade for the following studies; thereafter, the effect of adding a backward swept to the blade on the power production of the turbine was analyzed. The study will be carried on wind speeds in the range of 8 to $16 \mathrm{~m} / \mathrm{s}$, the range that the average of wind speed in most commercial wind farms falls within.

For each backward sweep configuration, two parameters have been examined: the sweep angle and the sweep start spanwise section. The definition of sweep angle is illustrated in Fig. 1. The sweep angle is defined by the angle between the baseline spanwise and the line connecting the root and the tip while the backward ratio is defined as the ratio between the tip digression and blade radius.

In this research, the backward ratio is considered $10 \%$, which starts at $50 \%$ spanwise section of each blade. Apparently, an optimization process can be run on backward swept to achieve the maximum amount of output power. The optimization process can be included an iterative loop, which includes four processes; geometric blade generation, mesh generation of the blade, CFD simulation and post-processing. The output power resulted from CFD calculations is used for modifying the geometrical parameters in a new iteration.

The same mesh topology used for the rotor with straight blades is applied here. The results were obtained by solving the RANS equations with the standard K- $\varepsilon$ as the turbulence model while the solution algorithm is SIMPLE. The results are obtained for the wind speed range between 8 to $16 \mathrm{~m} / \mathrm{s}$.

It is important to calculate the percentage of power increase due to the backward sweep. Table. 1 lists the percentage of computed power and thrust variation with respect to the original straight blade geometry in different wind speeds.

The results apparently reveal that not only the power increases with a backward swept blade but also the axial thrust decreases. In other words, a backward swept blade reduces aerodynamic loads on the rotor and increase blade efficiency. Backward swept blade is a better configuration for increasing the power at moderate and high wind speeds. This result is in agreement with the results obtained in Gaunaa et al, (2007) for winglet configuration.

It should be mentioned that the backward swept configuration is completely different from winglet and has a different aerodynamic effect on wind turbine performance.

The induced drag can be reduced by reducing the spanwise flow. The idea of adding a backward swept to the wind turbine blade is to produce a flow opposing the flow produced by the blade. This flow will tend to cancel or weaken the main flow of the blade and hence reduce the spanwise flow and consequently reduce the induced drag. The backward swept diffuses or spreads out the influence of the tip vortex and as a result it reduces the induced drag (Elfarra, 2011). 
Table. 1.

Comparison of power extraction between two rotors at different wind speeds calculated by CFD

\begin{tabular}{ccccccc}
\hline $\begin{array}{c}\text { Wind } \\
\text { speed } \\
(\mathrm{m} / \mathrm{s})\end{array}$ & $\begin{array}{c}\text { Power of } \\
\text { rotor with } \\
\text { straight blade } \\
(\mathrm{W})\end{array}$ & $\begin{array}{c}\text { Power of rotor } \\
\text { with backward } \\
\text { swept blade } \\
(\mathrm{W})\end{array}$ & $\begin{array}{c}\text { Percentage of } \\
\text { power } \\
\text { variation }\end{array}$ & $\begin{array}{c}\text { Thrust of rotor } \\
\text { with straight } \\
\text { blade } \\
(\mathrm{N})\end{array}$ & $\begin{array}{c}\text { Thrust of rotor } \\
\text { with backward } \\
\text { swept blade } \\
(\mathrm{N})\end{array}$ & $\begin{array}{c}\text { Percentage of } \\
\text { thrust variation }\end{array}$ \\
\hline 8 & 7548.33 & 5937.84 & -21.3 & 2423.02 & 1577.44 & -34.9 \\
9 & 10299.28 & 9270.92 & -10 & 2847.57 & 2021.44 & -29 \\
10 & 12870.43 & 12746.41 & -1 & 3236.66 & 2458.07 & -24.1 \\
11 & 14930.27 & 16111.26 & 7.9 & 3570.58 & 2871.05 & -19.6 \\
12 & 16471.01 & 19131.84 & 16.2 & 3864.81 & 3245.52 & -16. \\
13 & 17584.01 & 21651.73 & 23.1 & 4136.11 & 3577.07 & -13.6 \\
14 & 18341.65 & 23711.79 & 29.3 & 4386.37 & 3881.06 & -11.5 \\
15 & 18942.48 & 25377.06 & 34 & 4635.19 & 4167.53 & -10.1 \\
16 & 19590.77 & 26710.55 & 36.3 & 4895.96 & 4444.39 & -9.2 \\
\hline
\end{tabular}

To see the effect of the backward sweep, the output power and axial thrust were compared with the original blade at different wind speeds 8 to $16 \mathrm{~m} / \mathrm{s}$ in Fig. 5 and Fig. 6, respectively. These figures reveal that the increase in power due to this sweep retains a considerable value at higher wind velocities. In lower velocities extracted power from the rotor with swept blades is less than the original rotor, but by increasing the wind speed two curves become close to each other and after wind speed of approximately $10 \mathrm{~m} / \mathrm{s}$ the power extracted from the rotor with swept blades is higher than the original rotor. By increasing wind speed, this difference becomes bigger.

The backward sweep the had effect on the axial thrust decrease, which means that the power increase is arising from the reduction in the drag losses in moderate and high wind speeds. In Fig. 7, the wind velocity in the flow direction y (see Fig. 2), in both rotor with original blades and rotor with swept blades, has been compared in wind speed of $14 \mathrm{~m} / \mathrm{s}$. It can be clearly observed that the wind velocity in the flow direction is lower when the blades are swept especially behind the rotor, which means that a rotor with swept blades extracts more energy from the wind.

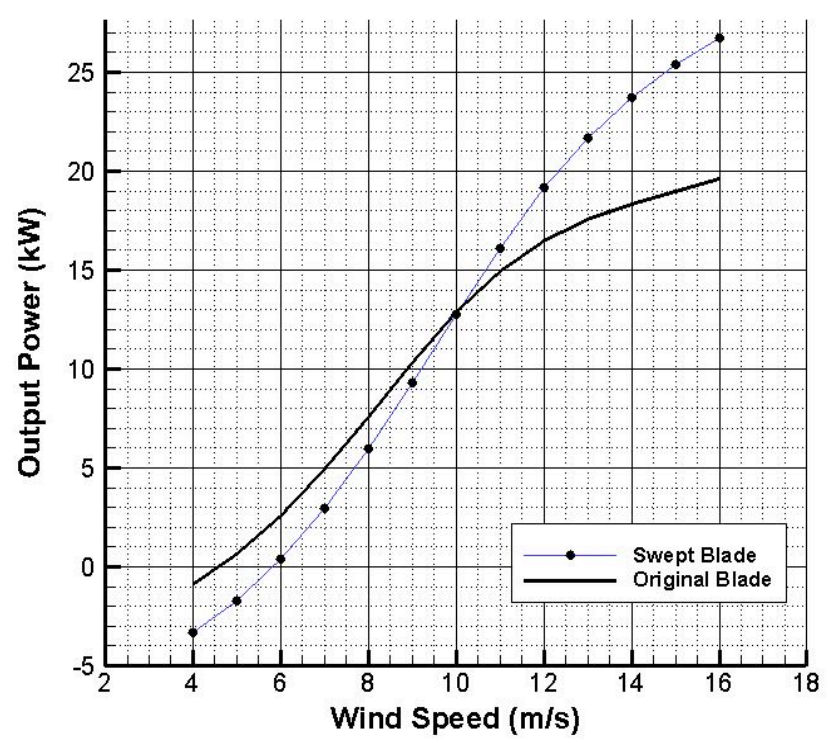

Fig. 5. Comparison of power extraction between two rotors at different wind speeds calculated by CFD

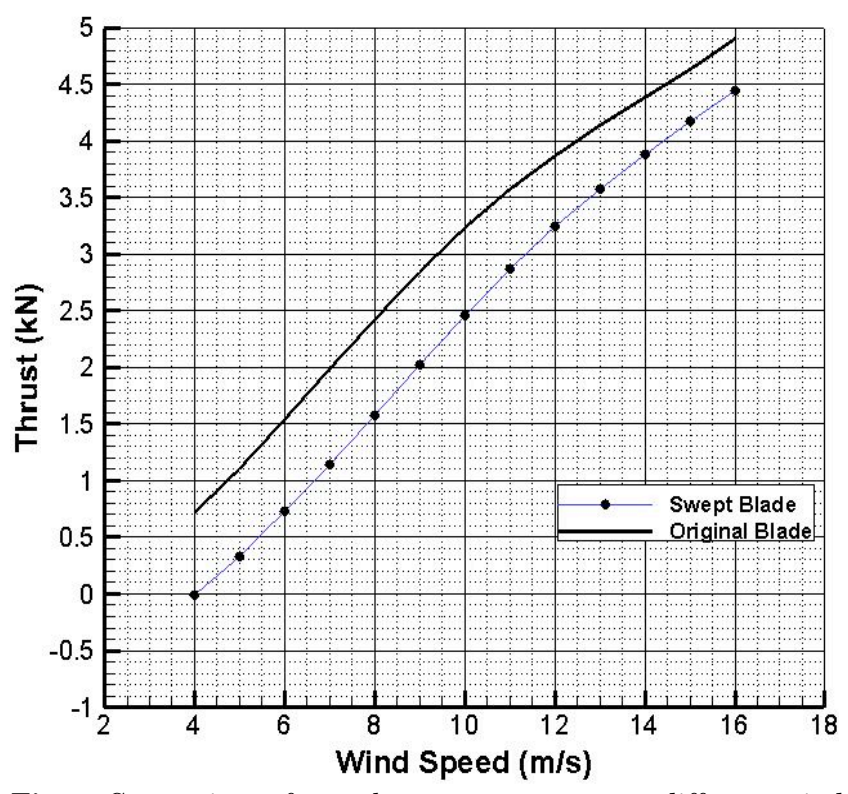

Fig. 6. Comparison of trust between two rotors at different wind speeds calculated by CFD

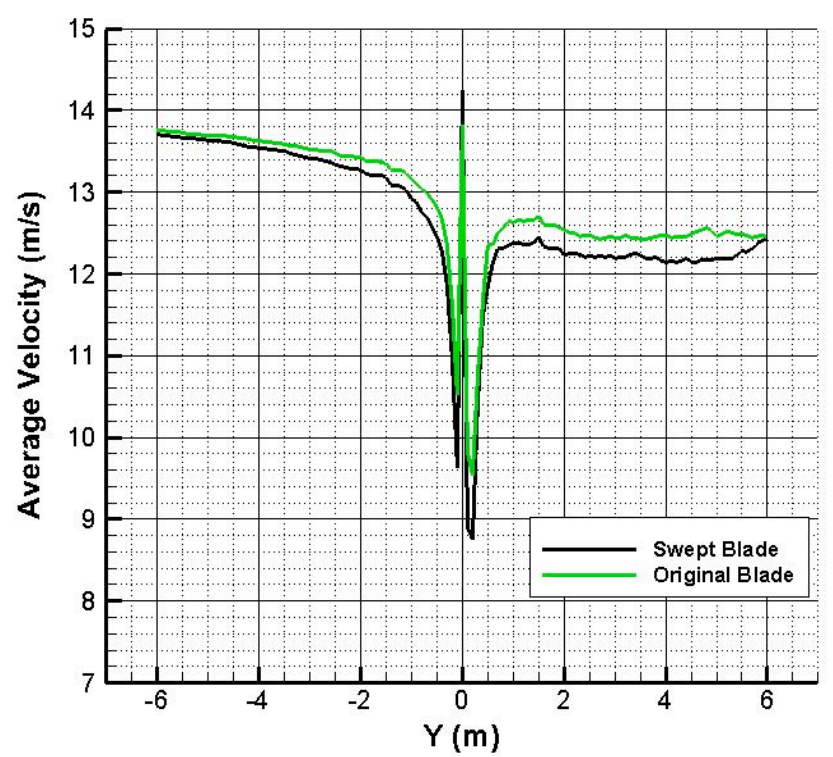

Fig. 7. Wind velocity comparison in flow direction 
Fig. 8 and Fig. 9 illustrate velocity magnitude and static pressure contours at three different spanwise sections, $20 \%, 50 \%$ and $80 \%$, at the wind speed of $5 \mathrm{~m} / \mathrm{s}$ respectively for original and swept blades. It is clear that the gradients of these flow properties at this wind speed for each original blade section are more extreme than related contours for swept blade.

Since the separation is very clear at higher speeds, the velocity magnitude and static pressure have been compared for both straight and swept blades in Fig. 10 and Fig. 11 respectively at three different spanwise sections, $20 \%, 50 \%$ and $80 \%$, at the wind speed of $15 \mathrm{~m} / \mathrm{s}$ to see the effect of the backward swept on the separation. It is clear that gradients at this wind speed are more intensive than the related gradients at lower speeds such as $5 \mathrm{~m} / \mathrm{s}$ which is illustrated above. Furthermore, flow separation for the original blade at each wind speed is more intensive in comparison of the swept blade. So, the backward swept has a clear and notable effect on changing the flow separation point and velocity field, which has been specifically observed in spanwise sections $50 \%$ and $80 \%$.

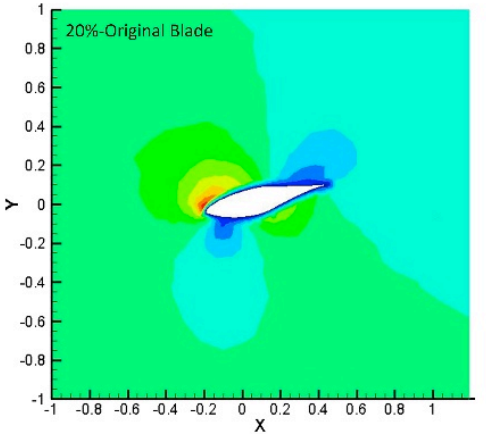

(a)

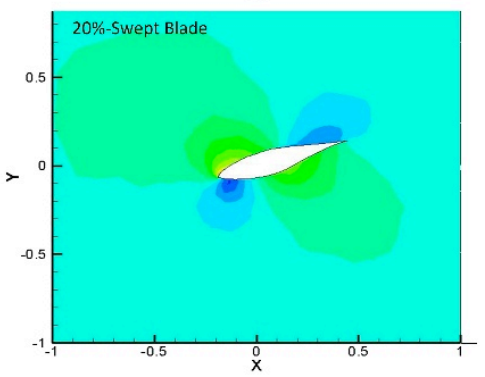

(d)

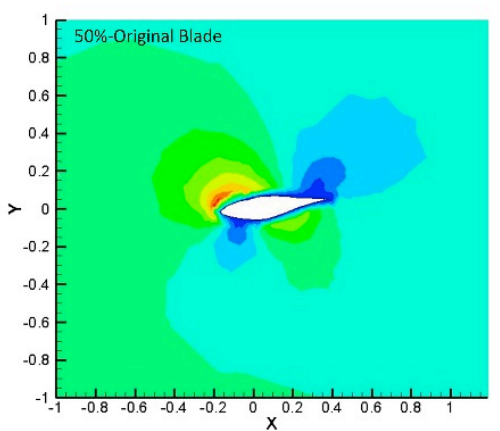

(b)

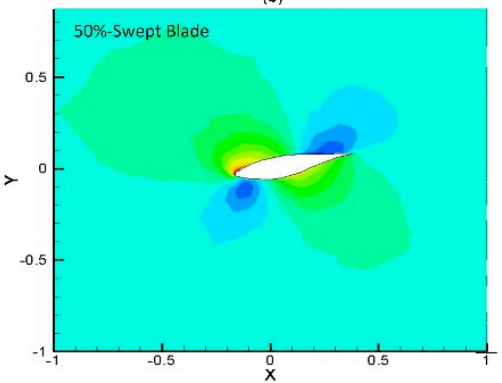

(e)
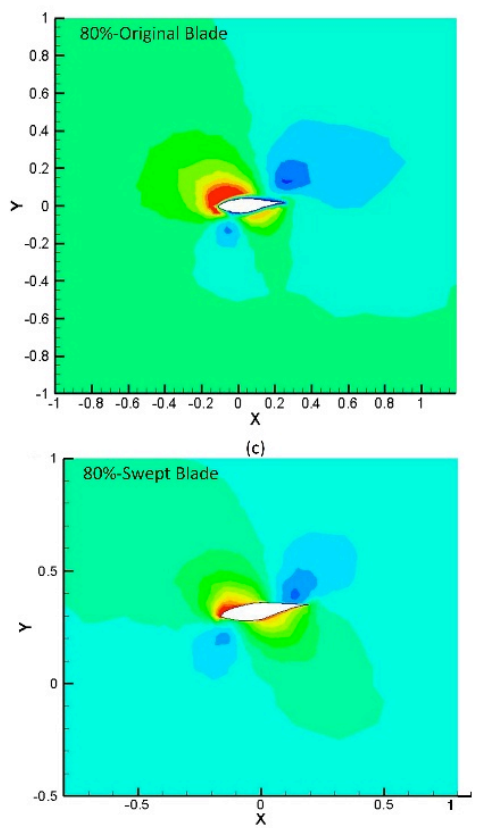

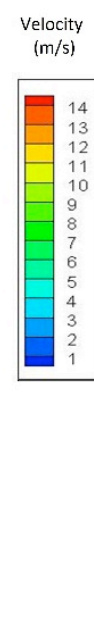

Fig. 8. Velocity magnitude contours comparison at the wind speed of $5 \mathrm{~m} / \mathrm{s}$ and different blades spanwise: a) $20 \%$ spanwise of original blade. b) $50 \%$ spanwise of original blade. c) $80 \%$ spanwise of original blade. d) $20 \%$ spanwise of swept blade. e) $50 \%$ spanwise of swept blade. f) $80 \%$ spanwise of swept blade
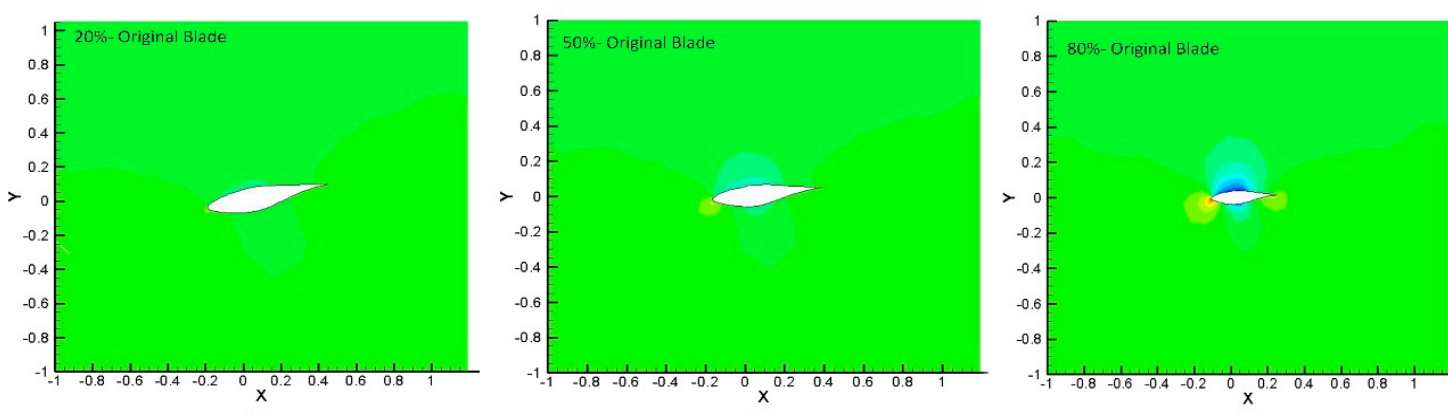

(b)
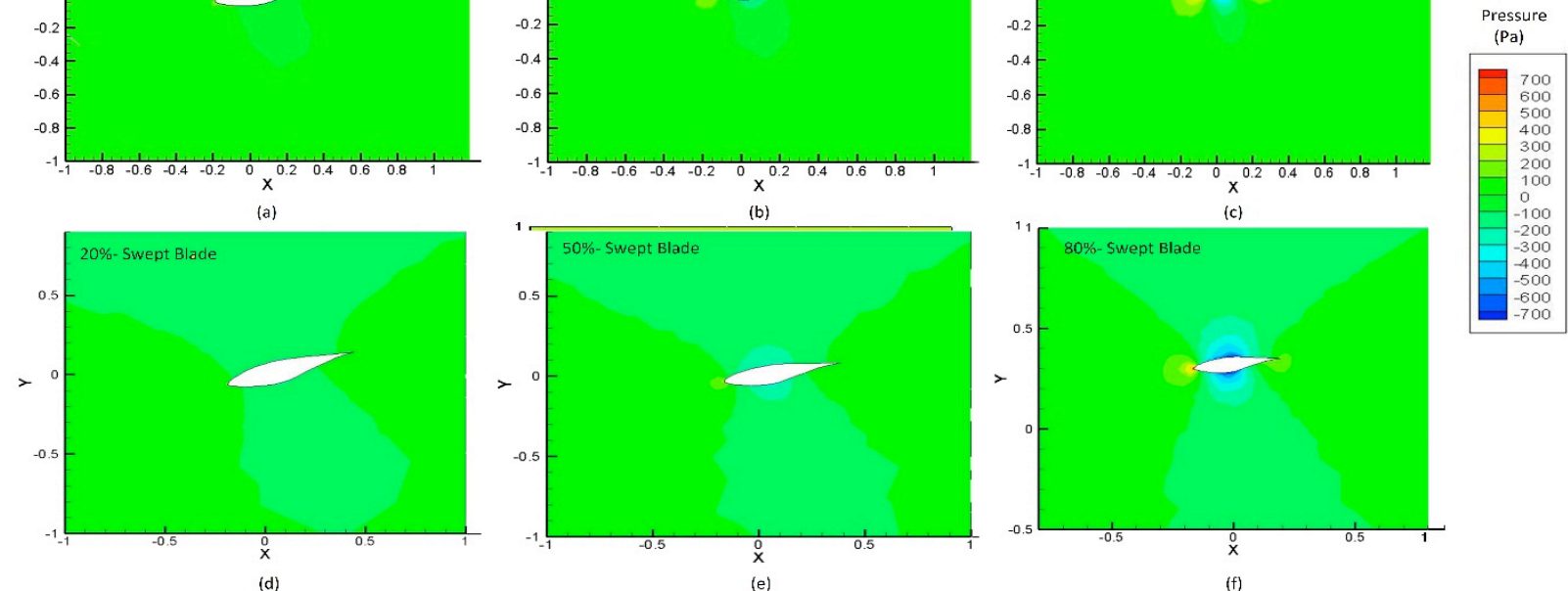

Fig. 9. Static pressure contours comparison at the wind speed of $5 \mathrm{~m} / \mathrm{s}$ and different blades spanwise: a) $20 \%$ spanwise of original blade. b) $50 \%$ spanwise of original blade. c) $80 \%$ spanwise of original blade. d) $20 \%$ spanwise of swept blade. e) $50 \%$ spanwise of swept blade. f) $80 \%$ spanwise of swept blade 


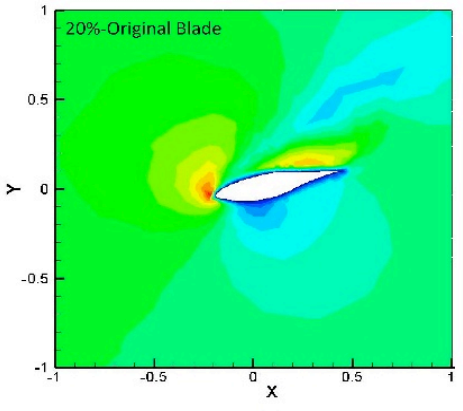

(a)

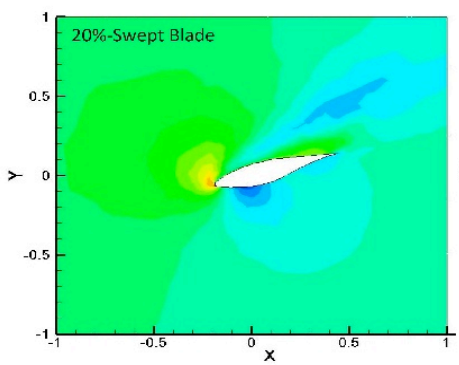

(d)

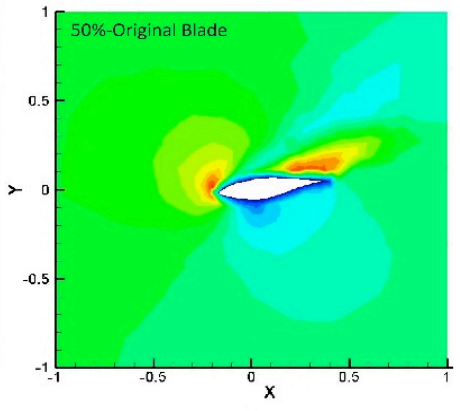

(b)

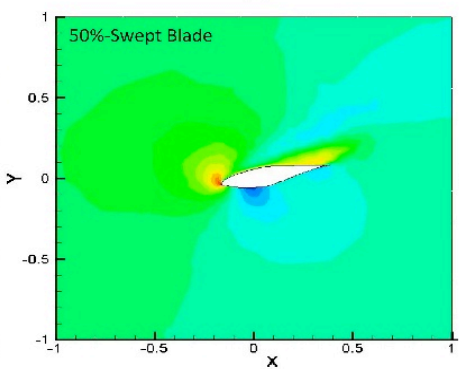

(e)

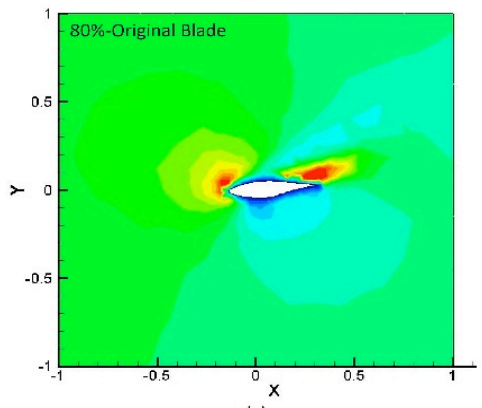

(c)

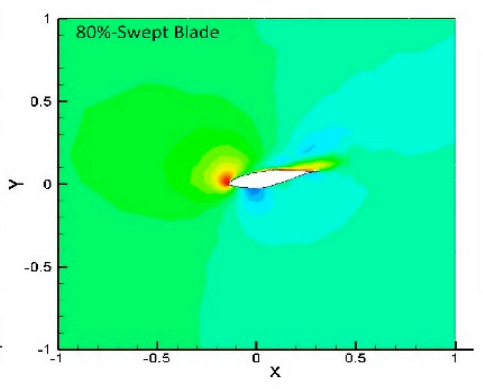

(f)

Fig. 10. Velocity magnitude contours comparison at the wind speed of $15 \mathrm{~m} / \mathrm{s}$ and different blades spanwise: a) $20 \%$ spanwise of original blade. b) $50 \%$ spanwise of original blade. c) $80 \%$ spanwise of original blade. d) $20 \%$ spanwise of swept blade. e) $50 \%$ spanwise of swept blade. f) $80 \%$ spanwise of swept blade

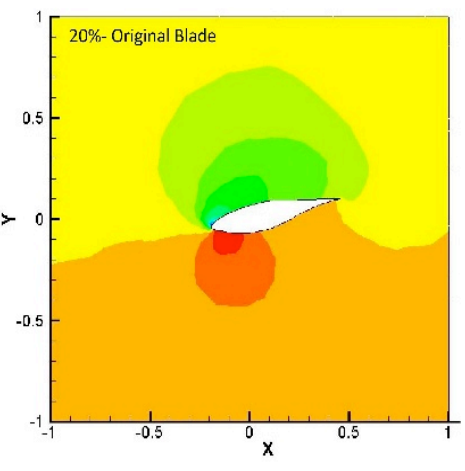

(a)

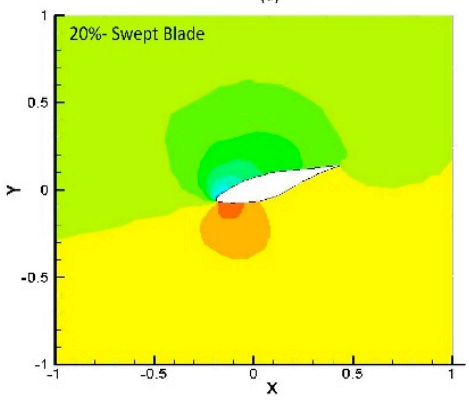

(d)

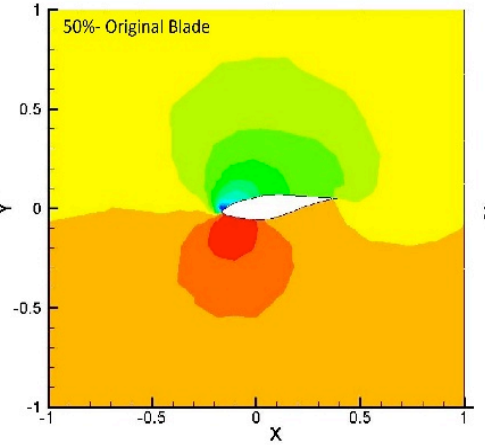

(b)

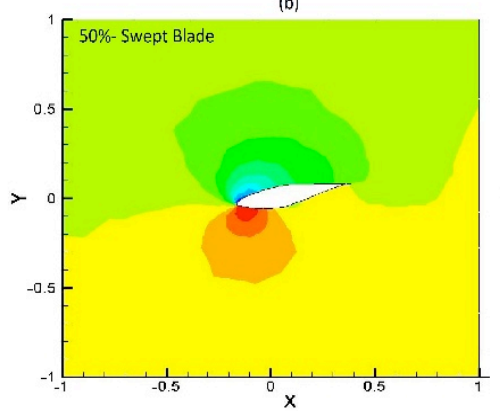

(e)

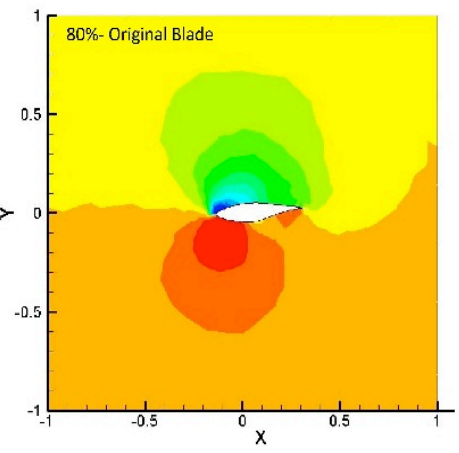

(c)

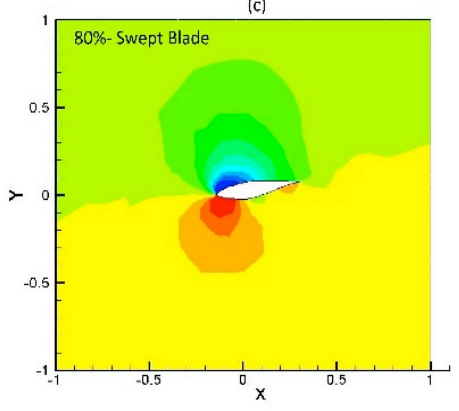

(f)

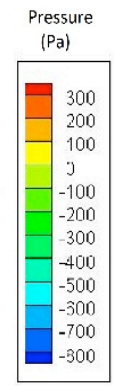

Fig. 11. Static pressure contours comparison at the wind speed of $15 \mathrm{~m} / \mathrm{s}$ and different blades spanwise: a) $20 \%$ spanwise of original blade. b) $50 \%$ spanwise of original blade. c) $80 \%$ spanwise of original blade. d) $20 \%$ spanwise of swept blade. e) $50 \%$ spanwise of swept blade. f) $80 \%$ spanwise of swept blade

Fig. 12 illustrates the trailing vortex comparison in the reign around the rotor at wind speeds of 5 and $8 \mathrm{~m} / \mathrm{s}$. Trailing vortex has been achieved from an Iso-Surface with constant vorticity magnitude. It is notable that the trailing vortex produced by the original blade is bigger than the one produced by swept blade. These results lead to the increase of torque and decrease of thrust on the rotor due to the swept blades, especially in the higher wind speeds.

\section{Conclusions}

The main purpose of this study has been implementing the aerodynamic design of a backward swept blade for horizontal axis wind turbine rotor blades, which has been already implemented to aircraft wings for the transonic cruise at Mach numbers.

A full-scale analysis on NREL VI 3-bladed 20kW wind turbine with full rotor modeling approach was carried out. Simulation results represent an acceptable 
agreement between the power output obtained from the full rotor approach and the available experimental results reported in the literature. In this way, our modeling was validated by comparing the CFD analysis results and available experimental data.

A backward swept blade has been considered and analyzed independently based on the CFD code. The results indicate that from an aerodynamic point of view, with the backward swept, the flow is more attached and directed to the downstream without separation. Because of this the power extraction can be increased with a more complicated geometry in higher wind speeds while the axial thrust decreases in all wind speeds that reduce adverse aerodynamic loads on the rotor.

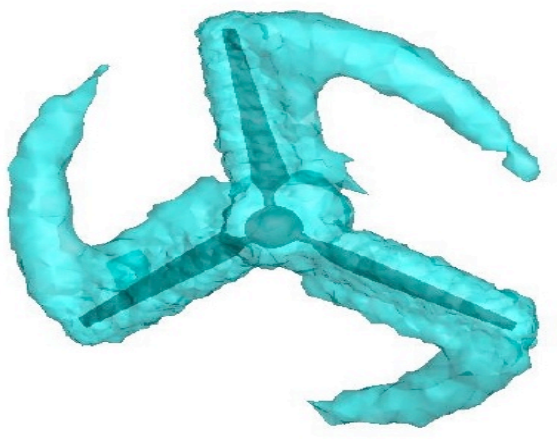

(a)

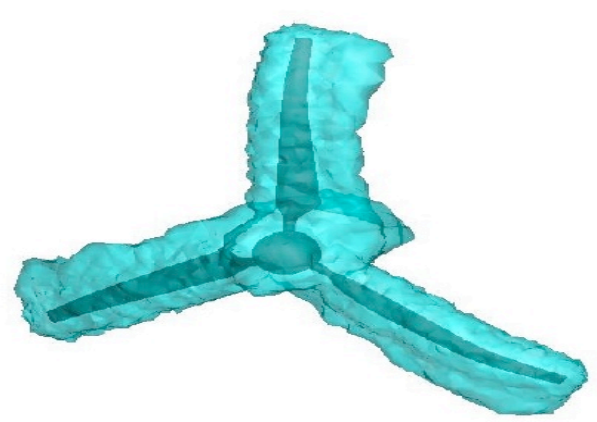

(c)
In the upcoming studies, an experimental test will hopefully make the results more reliable. Besides, further studies are required to optimize the sweep angle and start section of the sweep to achieve the maximum power output increase. A noise analysis can also show the effect of this sweep on noise production and environmental considerations. Studies about the aeroelasticity analysis of structural stability and strength of the blade due to the addition of a backward swept also are necessary to achieve a reliable method of extracting more power from wind turbines.

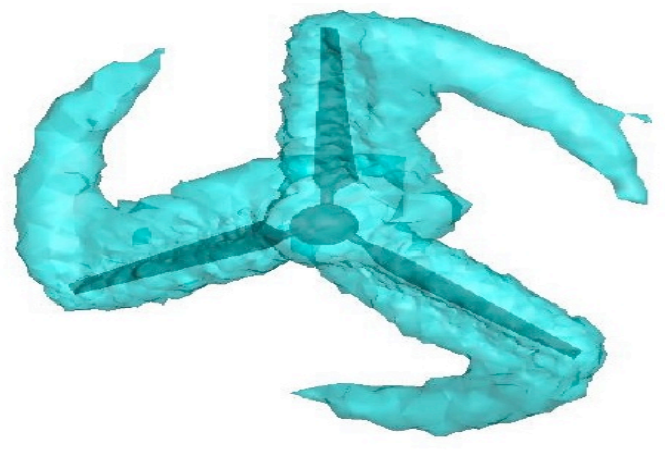

(b)

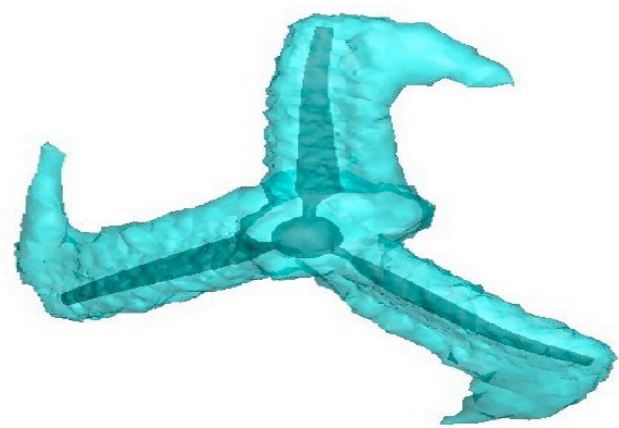

(d)

Fig. 12. Comparison of the trailing vortex between original and swept blades (a) straight blade at wind speed of $5 \mathrm{~m} / \mathrm{s}$ (b) straight blade at wind speed of $8 \mathrm{~m} / \mathrm{s}$ (c) Swept blade at wind speed of $5 \mathrm{~m} / \mathrm{s}$ (d) Swept blade at wind speed of $8 \mathrm{~m} / \mathrm{s}$

\section{References}

AbdelSalam, A. M., Ramalingam, V., (2014) Wake prediction of horizontal-axis wind turbine using full-rotor modeling, J. Wind Eng. Ind. Aerodyn., 124, 7-19.

Balduzzi, F., Bianchini, A., Maleci, R., Ferrara, G., Ferrari, L. (2016) Critical issues in the CFD simulation of Darrieus wind turbines, Renew. Energy, 85, 419-435.

Betz, A. (2014) Introduction to the theory of flow machines, Pergamon Press.

Cao, L., Ji, Y., Wang, Z., Yuan, W. (2012) Analysis on the influence of rotational speed to aerodynamic performance of vertical axis wind turbine, Procedia Eng., 31, 245-250.

Chattot, J. J. (2009) Effects of blade tip modifications on wind turbine performance using vortex model, Comput. Fluids, 38(7), 1405-1410.

Elfarra, M. A., (2011) Horizontal Axis Wind Turbine Rotor Blade: Winglet and Twist Aerodynamic Design and Optimization Using CFD, Middle East Technical University.

El-Wakil, M. M. (1984) Power plant Technology. McGraw-Hill International.
Farrugia, R., Sant,T., Micallef, D. (2016) A study on the aerodynamics of a floating wind turbine rotor, Renew. Energy, 86, 770-784.

Gaunaa, M., Johansen, J. (2007) Determination of the maximum aerodynamic efficiency of wind turbine rotors with winglets, Journal of Physics: Conference Series, 75, 012006.

Giguere, P., Selig, M. S. (1999) Design of a tapered and twisted blade for the NREL combined experiment rotor, NREL/SR, 500,26173

Janajreh, I., Qudaih, R., Talab, I., Ghenai, C. (2010) Aerodynamic flow simulation of wind turbine: downwind versus upwind configuration," Energy Convers. Manag., 51(8), 1656-1663.

Jeon, M., Lee, S. (2014) Unsteady aerodynamics of offshore floating wind turbines in platform pitching motion using vortex lattice method, Renew. Energy, 65, 207-212.

Li, Y., Paik, K. J., Xing, T., Carrica, P. M. (2012) Dynamic overset CFD simulations of wind turbine aerodynamics," Renew. Energy, 37(1),285-298. 
Make, M., Vaz, G. (2015) Analyzing scaling effects on offshore wind turbines using CFD, Renew. Energy, 83,1326-1340.

Mohamed, M. H., Ali, A. M., Hafiz, A. A. (2015) CFD analysis for $\mathrm{H}$-rotor Darrieus turbine as a low speed wind energy converter, Eng. Sci. Technol. Int. J., 18(1), 1-13.

Subramanian, B., Chokani, N., Abhari, R. S. (2016) Aerodynamics of wind turbine wakes in flat and complex terrains, Renew. Energy, 85, 454-463.
Sedaghat, A., Assad, M. E. H., Gaith, M. (2014) Aerodynamics performance of continuously variable speed horizontal axis wind turbine with optimal blades, Energy, 77, 752-759. 